\title{
NOISE SPECTROSCOPY OF RESISTIVE COMPONENTS AT ELEVATED TEMPERATURE
}

\author{
Adam Witold Stadler ${ }^{1)}$, Zbigniew Zawiślak ${ }^{1)}$, Andrzej Dziedzic ${ }^{2)}$, Damian Nowak ${ }^{2)}$ \\ 1) Rzeszów University of Technology, Powstanców Warszawy 12, 35-959 Rzeszów, Poland ( $₫$ astadler@prz.edu.pl, +48 17 865 1116) \\ 2) Wroctaw University of Technology, Wybrzeże Wyspiańskiego 27, PL 50-370 Wrocław, Poland (Andrzej.Dziedzic@pwr.wroc.pl)
}

\begin{abstract}
Studies of electrical properties, including noise properties, of thick-film resistors prepared from various resistive and conductive materials on LTCC substrates have been described. Experiments have been carried out in the temperature range from $300 \mathrm{~K}$ up to $650 \mathrm{~K}$ using two methods, i.e. measuring $(i)$ spectra of voltage fluctuations observed on the studied samples and (ii) the current noise index by a standard meter, both at constant temperature and during a temperature sweep with a slow rate. The $1 / f$ noise component caused by resistance fluctuations occurred to be dominant in the entire range of temperature. The dependence of the noise intensity on temperature revealed that a temperature change from $300 \mathrm{~K}$ to $650 \mathrm{~K}$ causes a rise in magnitude of the noise intensity approximately one order of magnitude. Using the experimental data, the parameters describing noise properties of the used materials have been calculated and compared to the properties of other previously studied thick-film materials.
\end{abstract}

Keywords: noise spectroscopy, low-frequency noise, resistance noise, low-frequency noise measurement, thick-film resistors

(C) 2014 Polish Academy of Sciences. All rights reserved

\section{Introduction}

Modern electronic devices and modules are the subject of a miniaturization trend. Apart from spectacular achievements in semiconductor integrated circuits production, where each year the characteristic size of the technology process is successively decreased according to Moore's law, substantial progress takes place also in passive component manufacturing. The latter is especially of great importance, as the number of passives fall for each active component rise, stimulating research and development effort in the field of new materials and technologies [1], like e.g. embedding passives into printed circuit boards [2], thick-film technologies that use both quite new materials and layer deposition methods, including various printing techniques [3, 4], in order to improve resolution and to reduce dimensions of the components as well as to develop new functional devices [1].

Evaluation of parameters of passives by means of noise measurements is an emerging technique. Although it is much more sophisticated, compared with other electrical quantity measurements, it may be a source of important knowledge concerning stability and reliability of electronic components [5-7]. Moreover, noise measurements are a very helpful diagnostic and research tool [8-12] that gives valuable information which could not be obtained by investigations of other electrical quantities, like e.g. ordinary tests of the components resistance. It is due to the fact that the noise measurements are much more sensitive to inhomogeneity of an internal structure of a resistive material, since power spectral density (PSD) of voltage fluctuations observed in a resistor (excited by a constant current) and conductance are proportional to the $4^{\text {th }}$ and $2^{\text {nd }}$ power of local current distributions, 
respectively. A good example are thick-film resistors (TFRs), where conductive and resistive materials interact in the termination/resistor body area. Using the noise measurement it is possible to gain more complete knowledge about phenomena that occurred at the interface area and its quality [7, 13], that could not be obtained using ordinary resistance measurements, since the contact resistance is often close to zero, provided that resistive and conductive materials were selected carefully [7]. Taking into account the above arguments it is clear that parameters describing noise properties are often used as a quality indicator of both materials and their interfaces [7, 13]. Furthermore, it is also possible to gain fundamental knowledge about noise sources and even their spatial distribution [7], what supports electrical transport models [14]. The importance of information deduced from experiments on noise properties of the electronic components is also of practical usefulness since it may be then used for the improvement of a manufacturing process to achieve technological advantage [7, 13]. It should be noted that noise is at the front of new technological improvements not only in micro- but also in nanotechnology including new layered [15] and porous materials as well as structures [16].

Despite of many advantages of electronic component characterization by means of noise measurements, it is still lacking thorough studies concerning noise in TFRs in a wide temperature range. Our earlier works [17] reported on experiments carried out in the range from room temperature down to $0.3 \mathrm{~K}$, what was achieved with the use of helium and nitrogen cryostats. On the other hand, there are only a few papers concerning studies of noise in TFRs at elevated temperature [18-20] (but for example the noise characterization of resistance gas sensors needs such measurements at significantly elevated temperature [21, 22]). In this paper, we report results of noise property studies of thick-film resistors made from various commercial materials deposited on LTCC substrates. Experiments were carried out in the temperature range from $300 \mathrm{~K}$ up to $650 \mathrm{~K}$, which is very important from the point of view of application in commercial electronics.

\section{Sample preparation}

The sample resistors have been deposited on LTCC substrates made of DP951 (DuPont) tape. On each substrate a set of three resistors of $200 \mu \mathrm{m}$ width and lengths of 30, 60, 90, 120, 150 , and $300 \mu \mathrm{m}$ has been prepared. Successive stages of the resistor preparation process include (i) the firing of the substrate, (ii) screen-printing of the conductive layer on the fired substrate, (iii) laser scribing of the dried conductive layer to define the length of the resistor, (iv) firing of the conductive layer, (v) screen-printing of the resistive paste into the incised place, and finally ( $v i$ ) firing of the resistive layer using a suitable temperature profile with $875^{\circ} \mathrm{C}$ peak firing temperature [23]. The thickness of the final resistive layers has been measured with the Talysurf CCI (from Taylor Hobson Precision) optical profilometer, giving $d \approx 15 \mu \mathrm{m}$ for the resistors of 30,60 , and $90 \mu \mathrm{m}$ length, and $d \approx 10 \mu \mathrm{m}$ for the others.

The conductive contacts were made of either Au-based ESL8844G (from ElectroScience Lab.) or PdAg-based DP6146 (DuPont) inks. The inks CF011, CF021, and CF041 (all from DuPont) of nominal sheet resistances, $R_{s q}, 10 \Omega / \mathrm{sq}, 100 \Omega / \mathrm{sq}$, and $10 \mathrm{k} \Omega / \mathrm{sq}$, respectively, were used as resistive films. Samples 8844CF011, 8844CF021, 6146CF011, 6146CF041 (such marks carry information both about conductive and resistive materials) have been investigated. Average room temperature sample resistances for each substrate have been gathered in Table 1. 


\section{Noise measurements}

Since we enter a new field of exploration, noise measurements have been carried out in two ways. Although there are known methods that analyze a noise signal in the time domain [24], we focus on the spectral analysis of fluctuations of an electrical quantity since it makes noise components and their evolution more apparent. Namely, the power spectral density function of voltage fluctuations arising in the sample was used for identification of noise components. The sample resistor has been placed in one of the bottom legs of the DC bridge, as shown in Fig. 1, while the upper legs were created by wire-wound resistors $R_{B}$, whose resistance was equal to $10 \mathrm{k} \Omega$ or $1 \mathrm{M} \Omega$, depending on the sample resistance $R_{\text {sample }}$, in order to fulfil the condition $\left.R_{B}\right\rangle R_{\text {sample. }}$ Resistance at room temperature of samples 6146CF041 was about $40 \mathrm{k} \Omega$, while for the others it was less than $200 \Omega$. In the other bottom leg of the bridge an adjustable wirewound resistor was inserted for bridge balancing. The voltage signal from the bridge diagonal was amplified in the differential low-noise preamplifiers (Signal Recovery model 5186) with a gain of 1000 and AC coupling, then low-pass filtered. Next, analog-to-digital conversion took place in a plug-in DAQ board. The cross-correlation method has been used in order to reject the interferences and noise introduced by the system $[6,25,26]$.

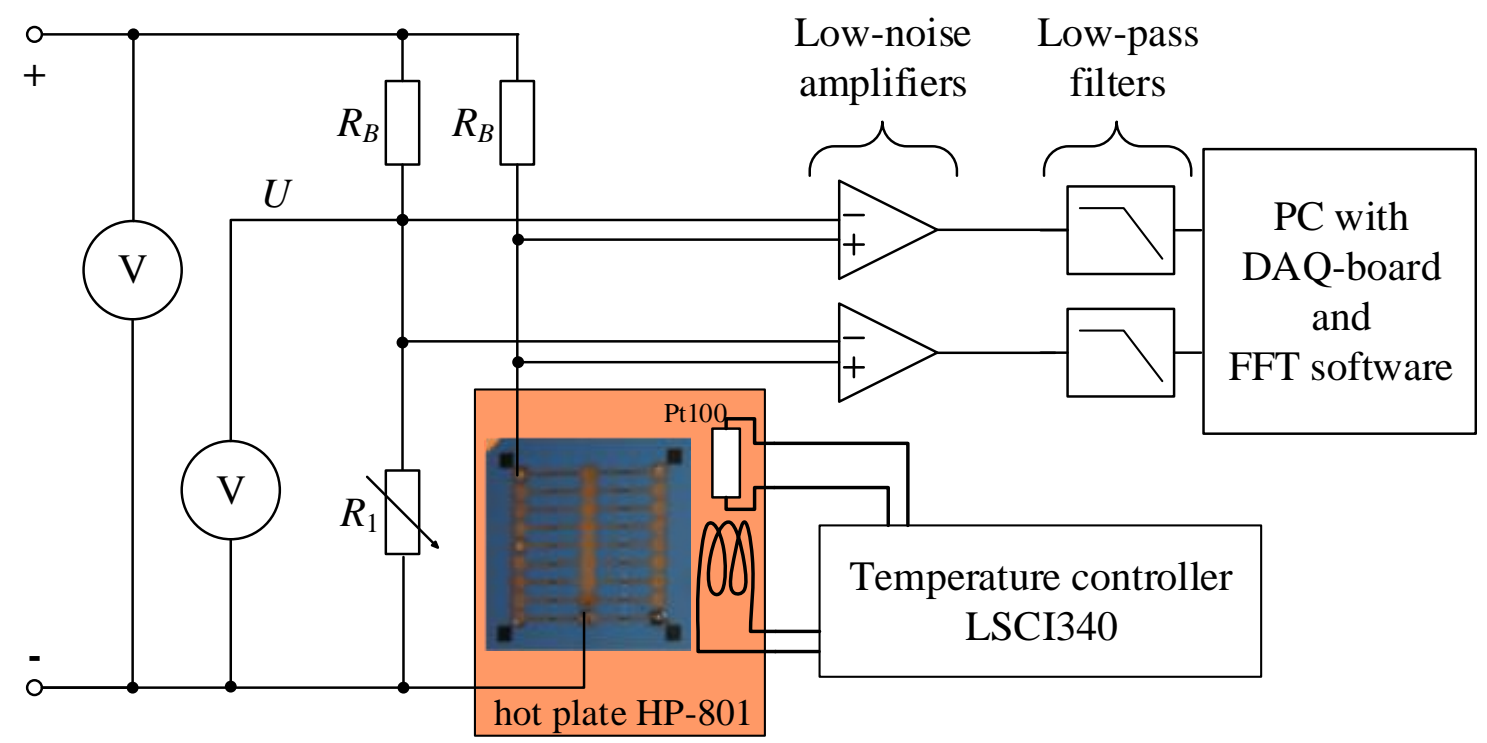

Fig. 1. DC bridge configuration used for noise spectra measurement at elevated temperature.

The cross-power spectral density $S_{V}(f)$ of voltage fluctuations was calculated using time records of $2 \mathrm{~s}$ duration. Consecutive spectra were averaged in real time, so that the total time of averaged spectra was $300 \mathrm{~s}$ and then average spectra were displayed on the PC screen and recorded. The cut-off frequency of low-pass filters used in the signal path was set to $30 \mathrm{kHz}$, while the sampling frequency in the DAQ board was $512 \mathrm{kHz}$. Only the low-frequency parts, up to $10 \mathrm{kHz}$, of the average spectra were recorded. The spectra were measured for different sample voltages, including $S_{V=0}$ for zero bias. The exemplary spectra of excess noise,

$$
S_{V e x}=S_{V}-S_{V=0},
$$

obtained for the sample 6146CF041 have been plotted in Fig. 3, where also $S_{V=0}$ has been added for reference. The equation (1) is the key point for calculation of excess noise and, as it is a subtractive method, it is the source of cumulative error apparent in the range of frequency 
and sample voltage where the total noise $\left(S_{V}\right)$ is close to the background noise, what is observed in Figs. 3a, and 5a.

Noise measurements at room temperature were carried out both for noise identification and selection of samples for further investigations. The next experiments were performed at higher temperature with the use of the hot plate HP-801 (Microtherm Wroclaw) with an embedded heater and a typical temperature sensor Pt100 connected to the temperature controller 340 (LakeShore Cryotronics). The main advantage of the controller is that apart from keeping a fixed temperature in the closed loop it may also force a temperature change with a programmed rate. However, due to the heater impedance mismatch (in the current model of the hot plate), temperature control in this set-up was limited only to $500 \mathrm{~K}$. Therefore, in order to reach even higher temperatures, up to $650 \mathrm{~K}$, an external high-voltage IZS 5/71 DC supply unit had to be involved in directly driving the heater.

Additional voltmeters shown in Fig. 1 monitor the sample bias, i.e. they directly measure the supply voltage of the bridge and the sample voltage. Then, having both voltages and a known value of $R_{B}$, sample current and sample resistance are calculated. Such configuration makes the simultaneous measurement of both noise spectra and sample resistance possible, what is very important during a temperature sweep.

The hardware part of the measurement setup was controlled by the software prepared in LabVIEW with the use of the virtual instrument (VI) concept [27]. VI was responsible for $(i)$ data acquisition from the DAQ-board, voltmeters and temperature controller, (ii) data processing in real time, including resistance and spectra calculation, and (iii) calculated data recording as well as presentation in graphs on computer screen. Post-experiment data analysis included excess noise spectrum extraction and its dependence on sample voltage evaluation in order to obtain parameters describing noise properties [28].

The second method applied for the noise properties evaluation was the use of the resistor noise test system Quan-Tech 315B, which gave values of current noise index (CNI) for different sample voltages [29]. The meter directly measures rms voltage in a frequency band of $1 \mathrm{kHz}$ width, centered geometrically at $1 \mathrm{kHz}$ for both biased and non-biased sample. Moreover, connecting the meter with the PC in order to control the time of averaging the output signals, a significant improvement of accuracy and a capacity has been achieved [30].

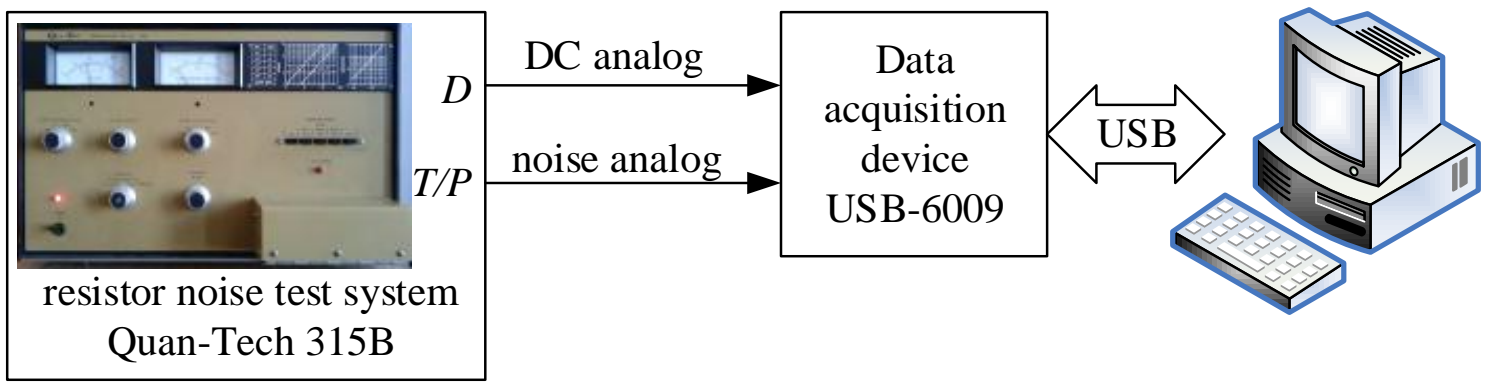

Fig. 2. System for computer monitoring of CNI measurements with the Quan-Tech 315B meter.

The sample resistors made of CF021 and CF041 inks were also studied previously [31], with the use of an AC technique (i.e. the bridge was supplied with an AC voltage source), which is certainly more sensitive (what is especially important for samples of small volume that need small excitation power) than the DC technique used in this work. In the $\mathrm{AC}$ technique observable spectra are not limited by the AC coupling in the signal path and therefore $S_{V}$ at frequencies as small as $30 \mathrm{mHz}$ might be measured, where $1 / f$ noise is more apparent opening the possibility of a significant reduction in the sample voltage. However, the main disadvantage of the AC technique is the need of keeping the bridge in balance. In this work, despite the fact that studies concern miniature thick-film resistors, what implies 
a limitation of sample excitation power, the DC technique was chosen, since only this configuration makes it possible to measure spectra during slowly-varying temperature with a controlled rate.

\section{Analysis}

\subsection{Noise components identification}

An exemplary set of excess noise spectra, for different sample voltages, obtained at room temperature for sample 6146CF041 has been plotted in Fig. 3a. It convinces that the $1 / f$ noise component is dominant. Hence, the product of frequency and $S_{V e x}$, averaged in a certain frequency band $\Delta f,\left\langle f S_{V e x}\right\rangle_{\Delta f}$, is a convenient measure of excess noise intensity. Such quantity, calculated from the experimental data for adjoining decade frequency bands, has been plotted in Fig. $3 \mathrm{~b}$ as a function of the sample voltage $U$ (points). Additionally, their approximations with $U^{2}$ for each decade have been added (lines) to demonstrate that the observed noise is caused by resistance fluctuations. It is worth to note that the applied method of estimation of noise intensity, based on averaging the product of frequency and excess noise over a certain frequency range, is reliable even for an extremely small value of sample voltage (e.g. $1.8 \mathrm{mV}$ for sample from Fig. 3), although part of the excess noise spectrum is below the background noise and therefore is significantly distorted due to the error introduced by eq. (1).
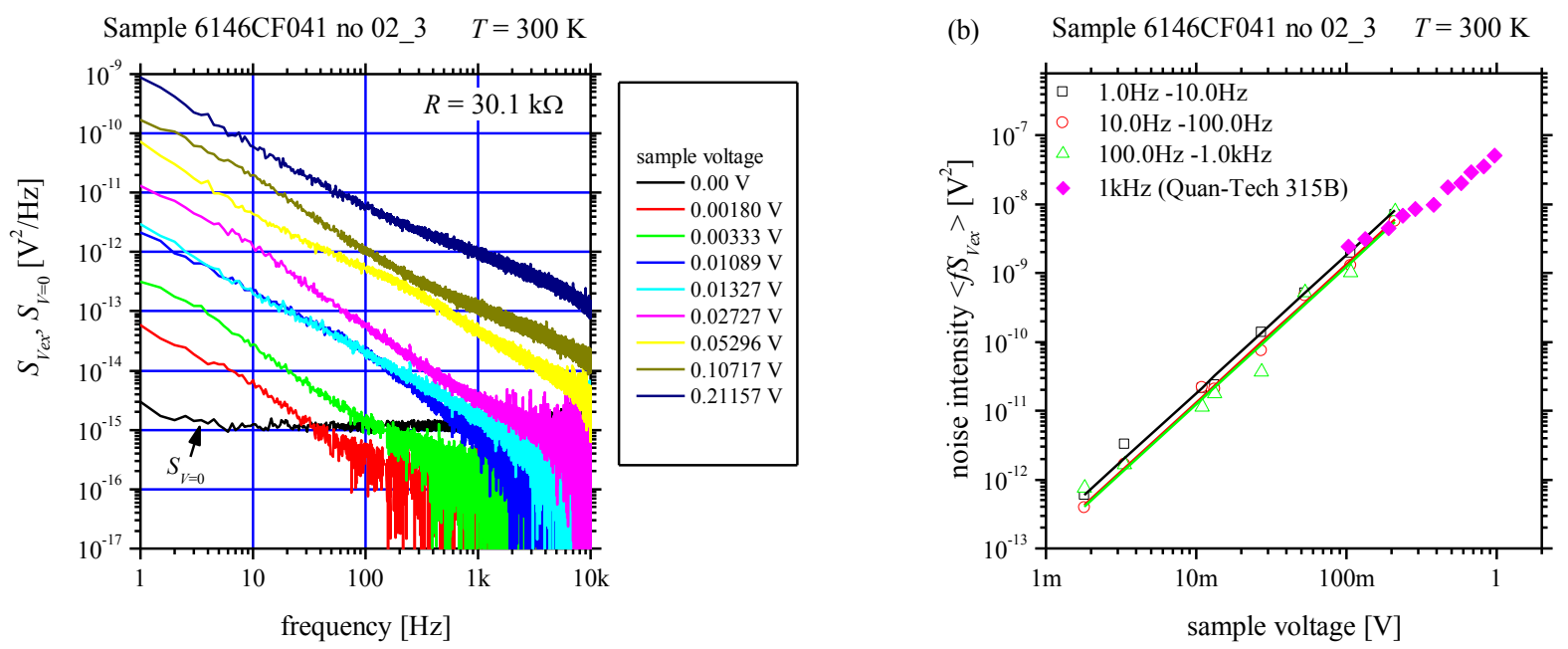

Fig. 3. (a) Spectra of excess noise, $S_{V e x}$, for sample $6146 \mathrm{CF} 041$ measured at room temperature for different sample voltages. (b) Noise intensities, calculated for decade frequency bands using data from Fig. 3a, plotted as a function of the sample voltage (open symbols) and their approximations (lines) with quadratic function. The noise intensity calculated from CNI has been added (solid symbols) for comparison.

The small discrepancy of points and approximating lines indicates that also other noise components exist. It is visible in Fig. 4, where the product $f S_{V e x} U^{-2}$, is plotted vs. frequency for two 6146CF041 samples of $30 \mu \mathrm{m}$ length. In such graphs pure $1 / f$ noise would be the set of overlapping horizontal lines (for different sample voltages). Therefore, other than $1 / f$ noise components may be brought up. Indeed, in Fig. 4 non-stationary noise components of Lorentzian shape have been revealed, that were hardly visible in the spectra shown in Fig. 3a. Another conclusion worth to note, deduced from Fig. 4, concerns levels of noise intensities, which differ approximately by 2 orders of magnitude for samples with the same geometry and on the same substrate. 
Approximations of noise intensities with the sample voltage squared, drawn in Fig. 3b, are of fundamental importance for further evaluation of the noise material intensity parameter,

$$
C \equiv\left\langle f S_{V e x}\right\rangle_{\Delta f} U^{-2} \Omega,
$$

where $\Omega$ is the sample volume. Its principal advantage is independence from the frequency, the sample voltage and sample volume. The value of parameter $C$ was extracted from the noise spectra as the product of the slope of the line approximating the noise intensity vs. sample voltage square dependence (see Figs. $3 \mathrm{~b}$ and $5 \mathrm{~b}$ ) and the sample volume. Moreover, taking into account the relation [6]:

$$
C N I=10 \log \left(10^{12} \sin 10\right) \text {, }
$$

where $s=\left\langle f S_{V e x}\right\rangle_{\Delta f} U^{2}=C / \Omega$, it is obvious that using the CNI meter it is also possible to obtain, equivalent to that from Fig. 3b, the relationship between the noise intensity and the sample voltage, what has been also proved in [30]. It is also shown in Fig. 3b where the data points calculated from the CNI values measured by the meter Quan-Tech 315B have been added for comparison (solid diamonds). The most important advantage of the CNI meter is the fast evaluation of sample properties with respect to $1 / f$ noise. On the other hand, the most troublesome disadvantage of this meter use is lack of information about noise components. Another one is the relatively large minimal excitation voltage, i.e. $0.1 \mathrm{~V}$, limiting the range of possible applications (see Fig. 3b). Furthermore, the meter is calibrated for pure $1 / f$ noise. Hence, the previous assurance that the $1 / f$ noise component is dominant, is necessary when using the CNI meter.

On the other hand, for thin-film materials the parameter $C_{u s}$ was introduced [32], which relates to $C$ by the sample thickness $d$, i.e. $C_{\text {us }}=C / d$. Moreover, there is an obvious relationship between the Hooge constant, $\alpha_{H}$, introduced in an empirical rule [33] for homogeneous materials so as $S_{V e x} \equiv \alpha_{H} U^{2} /\left(N_{C} f\right)$, where $N_{C}$ is the number of free carriers, and material noise intensity parameter $C$ used in this work; $\alpha_{H} / N_{C}=C \Omega$.

(a) Sample 6146CF041 no 02_3

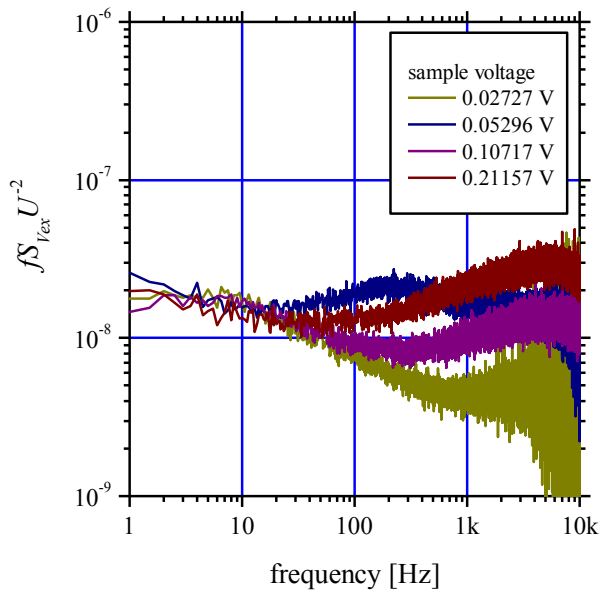

(b) Sample 6146CF041 no 02_2

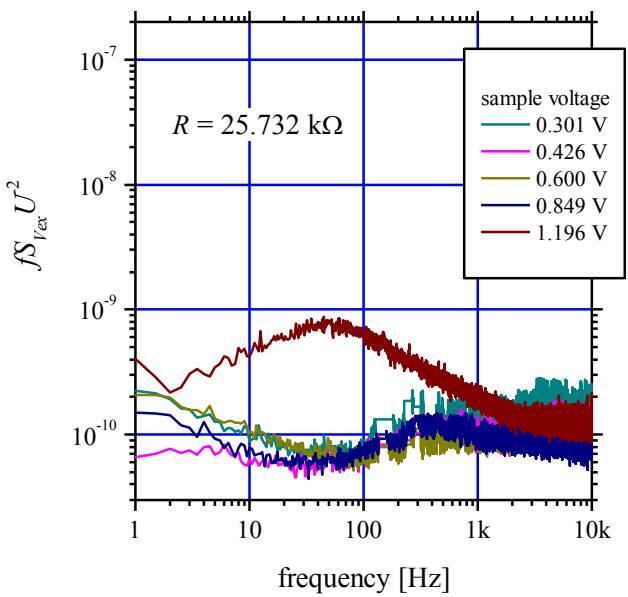

Fig. 4. (a) The product $f S_{V e x} U^{2}$, calculated using the data from Fig. 3a, plotted as a function of frequency for emphasizing other than $1 / f$ noise components.

(b) Product $f S_{V e x} U^{2}$ for another sample located on the same substrate.

Taking into account both the parameter $C$ and the resistivity $\rho=R_{s q} d$, another parameter is introduced for describing noise properties of resistive materials, $K \equiv C / \rho$, which is considered as a figure of merit and also the technology quality indicator with respect to $1 / f$ noise [32]. It is especially useful for the comparison of materials with significantly different resistivities. 
Averaged values of parameters obtained at room temperature for samples from each substrate have been gathered in Table 1 .

Table 1. Room temperature electrical and noise parameters describing the studied samples.

\begin{tabular}{||l|l|l|l|l||}
\hline \multirow{2}{*}{ Sample } & \multicolumn{4}{|c||}{ Parameter } \\
\cline { 2 - 5 } & $\rho[\Omega \mathrm{cm}]$ & $R_{s q}[\Omega]$ & $C\left[\mathrm{~m}^{3}\right]$ & $K\left[\mathrm{~m}^{2} / \mu \Omega\right]$ \\
\hline \hline $8844 \mathrm{CF} 011$ & 0.131 & 101.4 & $4.8 \cdot 10^{-26}$ & $4.3 \cdot 10^{-11}$ \\
\hline $8844 \mathrm{CF} 021$ & 0.178 & 140.0 & $8.6 \cdot 10^{-27}$ & $4.3 \cdot 10^{-12}$ \\
\hline $6146 \mathrm{CF} 011$ & 0.108 & 90.4 & $9.0 \cdot 10^{-26}$ & $8.4 \cdot 10^{-11}$ \\
\hline $6146 \mathrm{CF} 041$ & 594 & $396 \cdot 10^{3}$ & $1.8 \cdot 10^{-20}$ & $3.6 \cdot 10^{-09}$ \\
\hline
\end{tabular}

Analyzing data from Table 1, the discrepancy between nominal and real values of sheet resistance is apparent. Although, values of $R_{s q}$ for the layers made of CF011 and CF021 inks are close to each other, the significant difference is in the parameter $C$, whose value for CF021 is one order of magnitude smaller. On the other hand the layers of CF041 ink are definitely the most noisy. Resistors made of CF021 ink have been previously studied and a similar value of $C, C \approx 2 \cdot 10^{-27} \mathrm{~m}^{3}$ has been found [31], while for CF041 values in the range $(1-3) \cdot 10^{-25} \mathrm{~m}^{3}$ have been obtained depending on the sample size, what indicates much better noise properties than those observed for the samples studied in this work. However, the different technology process and large spread of $C$ values for samples made of CF041, which ranges from $4 \cdot 10^{-23} \mathrm{~m}^{3}$ to $6 \cdot 10^{-20} \mathrm{~m}^{3}$ while $R_{s q}$ ranges from 1.5 to $8.4 \cdot 10^{5} \Omega / \mathrm{sq}$, should be taken into account. Moreover, $R_{s q}$ for samples of CF041 ink studied in [31] ranged from 5 to $60 \mathrm{k} \Omega / \mathrm{sq}$, which was significantly different than that for our samples. Hence, rather parameter $K$ should be used for comparison of the samples with different resistivities. Its value for the components studied in [31] was $5 \cdot 10^{-13} \mathrm{~m}^{2} / \mu \Omega$ what is 4 orders of magnitude smaller (i.e. better) than that obtained in this work. However, it should be noted that among the studied samples only 6146CF041 were previously subjected to the thermal process, which took 160 hours at 200,300 , and $400{ }^{\circ} \mathrm{C}$, successively.

\subsection{Temperature dependence of noise}

Noise spectra, for selected samples, have been measured also at higher temperature, up to $650 \mathrm{~K}$. Typical noise spectra and the dependence of the noise intensity on the sample voltage calculated for the decade bands have been plotted in Fig. 5. It is visible that the $1 / f$ noise component still dominates and the dependence of its intensity on $U$ squared is valid. Using the experimental data, the parameter $C$ has been calculated for the frequency band $10 \mathrm{~Hz}-100 \mathrm{~Hz}$ and its dependence on temperature has been plotted in Fig. 6 (squares). For the samples from Fig. 6a and $6 \mathrm{c}$ the temperature rate was controlled and its value was kept at $1 \mathrm{~K} / \mathrm{min}$, while for sample from Fig. 6c measurements have been done during the unconstrained cooling from $475 \mathrm{~K}$ down to room temperature. 
(a) Sample 6146CF041 no 02_3 $T=450 \mathrm{~K}$

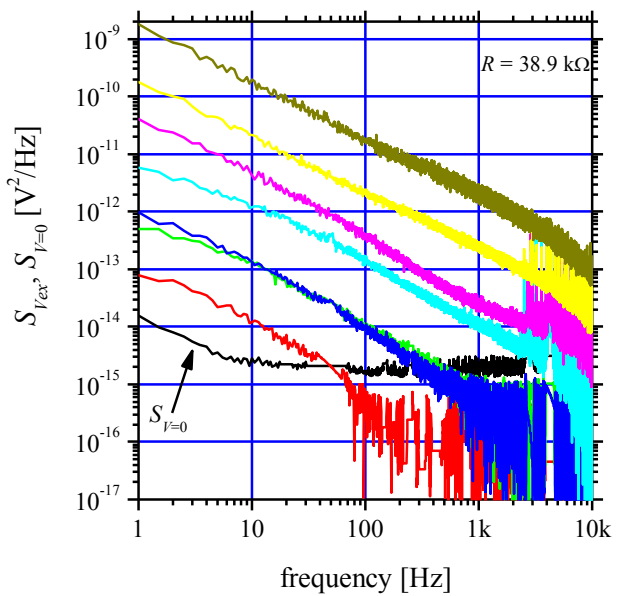

(b) Sample 6146CF041 no 02_3 $T=450 \mathrm{~K}$

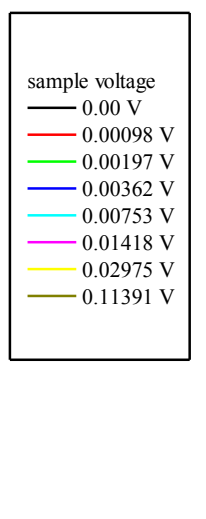

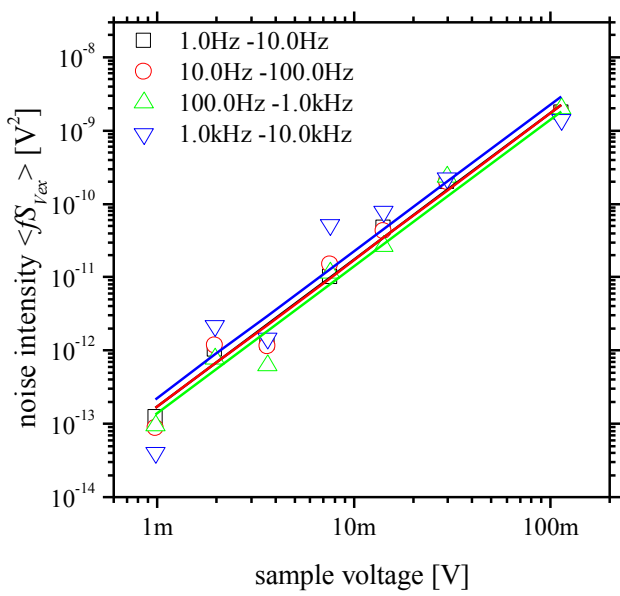

Fig. 5. (a) Excess noise spectra $S_{V e x}$ at $450 \mathrm{~K}$ for sample $6146 \mathrm{CF} 041$ for different sample voltages. (b) The noise intensity calculated for the decade frequency bands using the data from Fig. 5a vs. the sample voltage (open symbols) and their quadratic approximations (lines).

It has been observed for samples 6646CF041 that the temperature change from room temperature to $450 \mathrm{~K}$ causes a nearly 30 -fold increase in noise intensity and decrease in the sample resistance by $5 \%$. On the other hand, for sample $8841 \mathrm{CF} 021$ of $300 \mu \mathrm{m}$ length, the noise intensity in the same temperature range is increased 7 times and resistivity is decreased by $6 \%$ in a monotonic way. Furthermore, $S_{V e x}$ of the sample $8841 \mathrm{CF} 021$ has been investigated at fixed temperatures up to $650 \mathrm{~K}$, what reveals non-monotonic dependence of $C$ at the highest temperature, which has been depicted in Fig. 6c with solid circles. This result has been confirmed by the use of the CNI meter, what has been plotted with solid triangles in Fig. 6c, for reference.
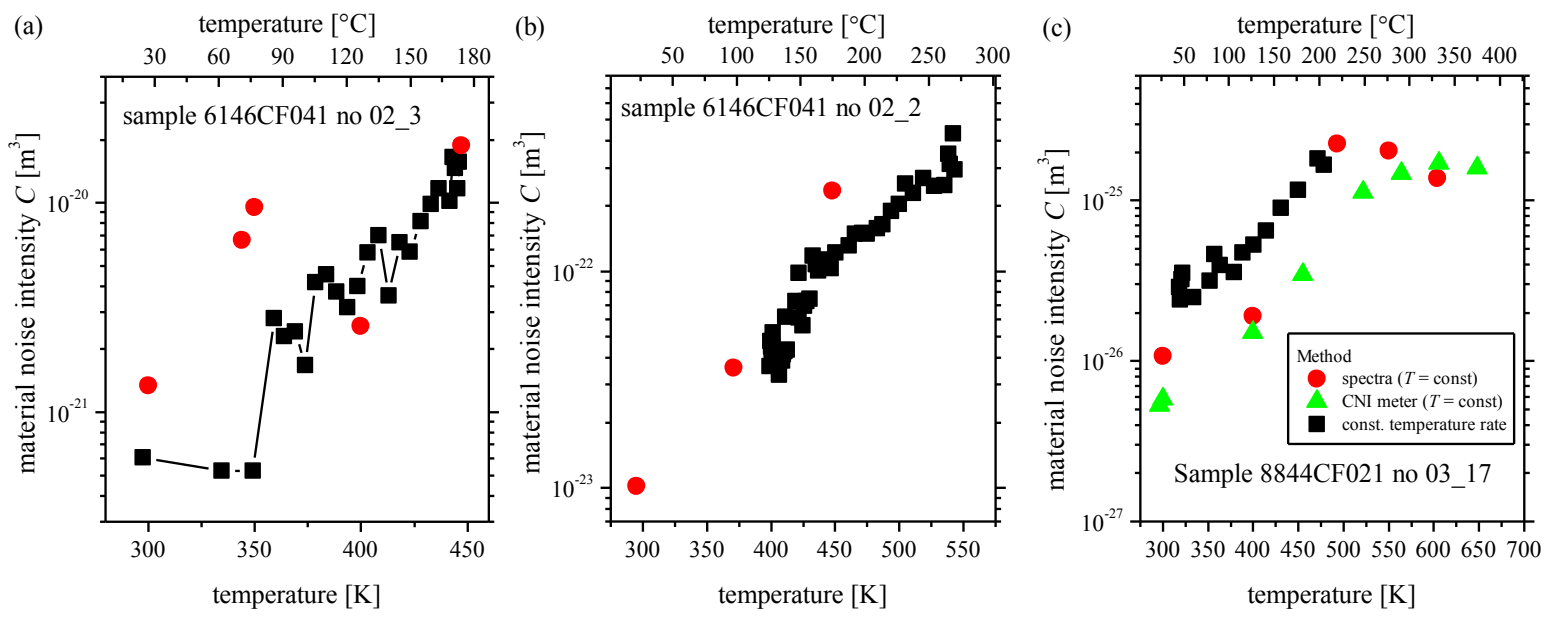

Fig. 6.The temperature dependence of the material noise intensity, the parameter $C$, for different samples, obtained in three ways: during the either controlled or free temperature sweep (squares), using both $S_{V e x}(f)$ (circles) and CNI (triangles) at the fixed temperature.

Temperature dependence of noise intensity in resistive materials was subject of only a few studies. For thick-film resistors based on bismuth ruthenate or lead ruthenate both monotonic and non-monotonic dependence of the noise intensity on temperature has been observed in the temperature range between $77 \mathrm{~K}$ and $650 \mathrm{~K}$ [19]. 


\section{Summary}

Results of studies on low-frequency noise carried out at room- and elevated temperature in thick-film microresistors made of various materials on LTCC substrates have been described. Noise properties have been evaluated in two ways: $(i)$ by the analysis of the cross-power spectral density function of voltage fluctuations in quasi-current excited samples in the DC bridge configuration, and (ii) by the use of the CNI meter. Both methods gave consistent results. Namely, $1 / f$ noise caused by resistance fluctuations, occurred to be the dominant component both at room- and elevated temperature, up to $650 \mathrm{~K}$. However, non-stationary noise components of Lorentzian shape of spectra have been also detected in some of the samples.

The material noise intensity parameter, $C$, has been calculated with the use of the experimental data, what confirmed excellent noise properties of the resistive layers made of CF011 and CF021 inks. The temperature dependence $C(T)$ has been also studied for selected samples, revealing a significant increase of the noise intensity when the temperature changes from $300 \mathrm{~K}$ to $450 \mathrm{~K}$. Moreover, for 1 sample $8841 \mathrm{CF} 02$, which was the subject of investigation even up to $650 \mathrm{~K}$, a non-monotonic dependence of $C(T)$ has been observed. However, it should be noted that the LTCC substrate is strongly susceptible to temperature, especially above $570 \mathrm{~K}[34]$.

As evidenced by the experiments, it is possible to use the noise spectroscopy technique with respect to samples of medium resistance $\left(R_{\text {sample }} \approx 160 \Omega\right.$ for the sample 8844CF021). However, studies carried out until now indicate that the accuracy of the noise spectra measurements should be improved, which is possible to do by applying a longer time of spectra averaging. It has been shown in [25], and also discussed in [35] that the averaging time plays a very important role in the averaging cross-spectra process as it decides about the effectiveness of removing unwanted components caused by uncorrelated noise sources. Furthermore, taking into account eq. (1), it is clear that improving the accuracy of both components $S_{V}$ and $S_{V=0}$ makes also excess noise more accurate, especially in the range of small sample voltage and higher frequencies. For example, in obtaining noise maps during noise spectroscopy experiments reported in [17] a time period of $600 \mathrm{~s}$ was used for averaging spectra collected during the temperature sweep with a slow rate. However a longer time of spectra averaging implies a lower rate of the temperature change what prolongs the experiment time, which took nearly 1 week for an individual noise map (i.e. obtaining one excess noise spectrum vs. temperature) in [17].

\section{Acknowledgements}

The work has been supported from Grant DEC-2011/01/B/ST7/06564 funded by the National Science Centre (Poland) and from Rzeszow University of Technology Project U-235/DS. Studies have been performed with the use of equipment purchased in the project No POPW.01.03.00-18-012/09 from the Structural Funds, The Development of Eastern Poland Operational Programme co-financed by the European Union, the European Regional Development Fund. 


\section{References}

[1] Bhattacharya, S.K., Tummala, R.R. (2000). Next generation integral passives: materials, processes, and integration of resistors and capacitors on PWB substrates, Journal of Material Science: Materials in Electronics, 11, 253-268.

[2] Dziedzic, A., Kłossowicz, A., Winiarski, P., Nitsch, K., Piasecki, T., Kozioł, G., Stęplewski, W. (2011). Chosen electrical properties and stability of passive components embedded in printed circuit boards, Electrotechnical Review, 87(10), 39-44.

[3] Parashkov, R., Becker, E., Riedl, T., Johannes, H.-H., Kowalsky, W., (2005). Large area electronics using printing methods, Proceedings of the IEEE, 93 (7), 1321-1329.

[4] Dziedzic, A. (2009). Modern thick-film and LTCC passives and passive integrated components, Inform. MIDEM (J. Microelectronics, Electronic Components and Materials), 39, 191-200.

[5] Dziedzic, A. (2002). Electrical and structural investigations in reliability characterisation of modern passives and passive integrated components, Microelectronics Reliability, $42,709-719$.

[6] Kolek, A. (2006). Experimental methods of low-frequency noise, University of Technology Publications, Rzeszow, Poland.

[7] Stadler, A. (2011). Noise properties of thick-film resistors in extended temperature range, Microelectronics Reliability, 51, 1264-1270.

[8] Rocak, D., Belavic, D., Hrovat, M., Sikula, J., Koktavy, B., Pavelka, J., Sedlakova, V. (2001). Low-frequency noise of thick-film resistors as quality and reliability indicator. Microelectronics Reliability; 41, 531-542.

[9] Jevtić, M.M. (1995) Noise as a diagnostic and prediction tool in reliability physics. Microelectronics Reliability, $35, \overline{455}-477$.

[10] Jevtić, M.M., Mrak, I., Stanimirović, Z. (1999). Thick-film quality indictor based on. noise index measurements. Microelectronics Journal; 30. 1255-1259.

[11] Zarnik, M.S., Belavic, D., Sedlakova, V., Sikula, J., Kopecky, M., Sedlak, P., Majzner, J. (2013), Comparison of the intrinsic characteristics of LTCC and silicon pressure sensors by means of $1 / f$ noise measurements, Radioengineering, 22 (1), 227-232.

[12] Bobalo, Y., Kolodiy, Z., Stadnyk, B., Yatsyshyn, S. (2013). Development of noise measurements. part 3. Passive method of electronic elements quality characterization, Sensors and Transducers, 152, 164-168.

[13] Mleczko, K., Zawiślak, Z., Stadler, A.W., Kolek, A., Dziedzic, A., Cichosz, J. (2008). Evaluation of conductive-to-resistive layers interaction in thick-film resistors, Microelectronics Reliability, 48, 881-885.

[14] Stadler, A.W., Kolek, A. (2007). Numerical simulations of low-frequency noise in $\mathrm{RuO}_{2}$-glass films, Proc. SPIE 6600, 66000Q.

[15] Balandin, A.A. (2013). Low-frequency $1 / f$ noise in graphene devices, Nature Nanotechnology, 8(8), 549-555.

[16] Granqvist, C.G., Green S., Jonson, E.K., Marsal R., Niklasson, G.A., Roos, A., Topalian, Z., Azens, A., Georén, P., Gustavsson, G., Karmhag, R., Smulko, J., Kish, L.B. (2008), Electrochromic foil-based devices: Optical transmittance and modulation range, 
effect of ultraviolet irradiation, and quality assessment by $1 / f$ current noise, Thin Solid Films, 516(17), 5921-5926.

[17] Kolek, A., Stadler, A.W., Ptak, P., Zawiślak, Z., Mleczko, K., Dziedzic, A. (2008). Noise and switching phenomena in thick-film resistors, J. Phys. D: Applied Physics, 41,025303 .

[18] Masoero, A., Rietto, A.M., Morten, B., Prudenziati, M. (1983). Excess noise and its temperature dependence in thick-film (cermet) resistors, J. Phys. D, 16, 669-674.

[19] Chen, T.M., Su, S.F., Smith, D. (1982). 1/f noise in Ru-based thick-film resistors, Solid-State Electron., 25, 821-827.

[20] Pellegrini, B., Saletti, R., Terreni, P., Prudenziati, M. (1983). 1/f' noise in thick film resistors as an effect of tunnel and thermally activated emissions, from measures versus frequency and temperature, Phys. Rev. B, 27, 1233-1243.

[21] Dziedzic, A., Kolek, A., Licznerski, B.W. (1999). Noise and nonlinearity of gas sensors - preliminary results, Proc. $22^{\text {nd }}$ int. Spring Seminar on Electronics Technology, Dresden-Freital (Germany), 99-104.

[22] Kotarski, M., Smulko, J. (2009). Noise measurement set-ups for fluctuations-enhanced gas sensing, Metrology and Measurement Systems 16, 457-464.

[23] Nowak, D., Miś, E., Dziedzic, A., Kita, J. (2009). Fabrication and electrical properties of laser-shaped thick-film and LTCC microresistors, Microelectronics Reliability 49 , $600-606$.

[24] Stawarz-Graczyk, B., Dokupil, D., Flisikowski, P. (2010). A method of RTS noise identification in noise signals of semiconductor devices in the time domain, Metrology and Measurement Systems, 17, 95-107.

[25] Sampietro, M., Fasoli, L., Ferrari, G. (1999). Spectrum analyzer with noise reduction by cross-correlation technique on two channels, Review of Scientific Instruments, 70(5), 2520-2525.

[26] Kolek, A., Ptak, P., Mleczko, K., Wrona, A. (2001). A further improvement of the measuring technique of bulk and contact components of resistance noise, Proc. $16^{\text {th }}$ Int. Conf. Noise in Physical Systems and 1/f Fluctuations, ICNF 2001, Gainesville, Florida, USA, 713-716.

[27] Stadler, A.W. (2007). Noise signal analyzer for multi-terminal devices, Proc. $31^{\text {st }}$ Int. Conf. of IMAPS-CPMT Poland Chapter, Rzeszów - Krasiczyn, Poland, 413-416.

[28] Stadler, A. (2011). Virtual instruments in low-frequency noise spectroscopy experiments, Proc. $35^{\text {th }}$ Int. Conf. of IMAPS-CPMT Poland Chapter, Gdańsk-Sobieszewo, 311-316.

[29] Test Method Standard Electronic and Electrical Component Parts, MIL-STD-202G, 2002.

[30] Stadler, A.W., Zawiślak, Z., Dziedzic, A., Stęplewski, W. (2012). Studies of noise in polymer thick-film resistors embedded in printed circuit boards, In Microelectronic Materials and Technologies, vol. 1, 82-97, Koszalin Technical University Monograph Series, Monograph No. 231.

[31] Kolek, A., Ptak, P., Dziedzic, A. (2003). Noise characteristics of resistors buried in low-temperature co-fired ceramics, J. Phys. D: Applied Physics, 36, 1009-1017. 
[32] Vandamme, L.K.J., Casier, H.J. (2004). The 1/f noise versus sheet resistance in poly-Si is similar to poly-SiGe resistors and Au-layers. Proc. of $34^{\text {th }}$ European Solid-State Device Research Conf. Leuven, Belgium, 21-23.

[33] Hooge, F. N.(1969), 1/f noise is no surface effect, Phys. Lett., 29A, 139-140.

[34] Unger, M., Fleig, J., Ahrens, M., Smetana, W., Radosavljevič, G. (2010). Permittivity and conductivity of various LTCC-tapes at high temperatures, Proc. $33^{\text {rd }}$ Int. Spring Seminar on Electronics Technology, 93-98.

[35] Giusi, G., Scandurra, G., Ciofi C. (2013). Estimation errors in 1/f' noise spectra when employing DFT spectrum analyzers, Fluctuation and Noise Letters, 12(1), 1350007. 